\title{
Immunization Guidelines for Children with Cancer in India
}

\author{
SANJAY Verma AND DEEPAK BANSAL * \\ Department of Pediatrics, Postgraduate Institute of Medical Education and Research, Chandigarh, India. \\ *deepakbansaldr@gmail.com
}

$\mathrm{T}$ The survival of children with cancers is improving, as noticeable in day-to-day clinical practice in low- and middle-income countries, including India. However, the task of the pediatric oncologist is not complete with the end of the specific chemotherapy regimen. A follow-up after the end of treatment for cancer is necessary not only for early detection of relapse, or treatment-related late effects, but also to ensure that the survivor can integrate into the society, and handle the infectious challenges that are a part of everyday living.

Missed vaccination opportunities in children treated for cancer could be because of a lack of knowledge among clinicians about the correct vaccination schedule for them [1]. The guidelines of societies from high-income countries may not be directly applicable to India due to several reasons, including the epidemiology of the disease, a varied national immunization schedule, availability of the vaccines and cost. Indeed, there was a long-awaited need to have immunization guidelines for children with cancer in India. Prevention of vaccinepreventable diseases in children receiving chemotherapy or who have completed chemotherapy is a challenging subject. On the one hand, there is a pressing need to prevent infections in this vulnerable population; on the other hand, limitations include a relatively restricted disease-burden and a lack of robust evidence for generating guidelines. It is in this context that the Pediatric Hematology-Oncology Chapter and the Advisory Committee on Vaccination and Immunization Practices of the Indian Academy of Pediatrics deserves a pat on the back for compiling the recommendation for immunization of children with cancer in India, published in this issue of Indian Pediatrics [2].

Before establishing the best strategies for immunization in children with cancer, it is essential to have an understanding of the alterations in the immune system of these patients. Although cancer itself can result in a variable degree of immune suppression, the primary etiology of immune suppression in these children is the cytotoxic chemotherapy, causing myelosuppression [3]. The recovery of cellular immunity following chemotherapy takes longer than that of humoral immunity. The duration for the reconstitution of the immune system following chemotherapy is variable and up to 3-12 months [3].

Live vaccines are typically contraindicated during and up to 6 months following the completion of chemotherapy. Killed vaccines are also best administered after 6 months from the end of treatment. Any killed vaccine, if administered during chemotherapy or before completion of 6 months following chemotherapy, should not be counted as an effective dose. Administration of the annual inactivated influenza vaccine is recommended during chemotherapy. Hepatitis B vaccine is administered for previously unimmunized children as per the suggested schedule. Newly diagnosed children with cancer are to receive pneumococcal vaccines as per age (PCV13 and PCV23), if not administered earlier. Oral polio vaccine is avoided in the siblings, while their remaining immunization can proceed without interruption. Contacts, including siblings, should be encouraged for varicella and annual influenza vaccination [4-6].

The critical clinical question is whether there is indeed a need for revaccination following chemotherapy when the primary immunization has been administered previously in childhood? This question cannot be adequately answered because the available data are controversial and conflicting. Three possible strategies have been reported: $(a)$ administration of a booster vaccine if there is evidence of low protective antibody titers, $(b)$ revaccination without assessing the residual immunity, or (c) continuing the regular childhood immunization schedule. Zignol, et al. [7] support the first strategy. They evaluated the serum titers of antibodies for tetanus, polio, diphtheria, hepatitis B virus (HBV), measles, mumps and rubella after chemotherapy in 192 children with solid tumors or leukemia. They observed low serum antibody titers in $52 \%$ of patients, mostly to HBV (46\%), followed by rubella, mumps, and measles 
(approximately 25\%). The administration of a booster dose at 6-12 months after the completion of chemotherapy was suggested as a cost-effective and straightforward way to restore humoral immunity [7].

Floredda, et al. [8] evaluated the titers of antibodies for HBV and tetanus in 70 children treated for leukemia at a median duration of 12 months after chemotherapy. They observed protective antibody titers comparable to those in healthy children in $85 \%$ of the patients. They recommended the regular childhood vaccination schedule to be continued as per the patient's age [8]. Law, et al. [9] dispute these findings; in their experience, the loss of protective antibodies after the end of chemotherapy was considerable in children with cancer, and revaccination at the earliest opportunity was recommended to be the best strategy.

The opinion regarding revaccination strategy for children with cancer after chemotherapy varies among research groups [10]. Limited reports on the risk of infections with Haemophilus influenzae, Streptococcus pneumoniae, Measles and Rubella are available [11,12]. A few experts might argue for obtaining antibody titers before recommending universal revaccination. However, the interpretation of antibody titers has limitations. The laboratory assessment of residual immunity for various diseases is not practical and cost-effective [10]. The simplified practice guidelines are indeed good for day-today practice; however, they are not entirely based on robust evidence. Studying and understanding the need for revaccination in patients receiving cancer chemotherapy remains a critical area for research.

Information regarding few vaccines provided in the consensus guidelines [2] needs to be revised as per the updated national immunization program. A dose of TT has been replaced with Td at 10 and 15 years of age, from 2019 onwards. Hib vaccine (as a component of pentavalent vaccine) is included in the national immunization program nationwide since 2015. Rotavirus vaccine and pneumococcal vaccine are also being administered in several Indian states under the national immunization program, and will be expanded.

Funding: None. Competing interests: None stated.

\section{REFERENCES}

1. Arora RS, Prabha S, Moulik NR, Bagai P. A survey of immunization practices in children with cancer in India. Pediatr Hematol Oncol J. 2016;1:2-5.

2. Moulik NR, Mandal P, Chandra J, Bansal S, Jog P, Sanjay S, et al. Immunization of Children with Cancer in India Treated with Chemotherapy - Consensus Guideline from the Pediatric Hematology-Oncology Chapter and the Advisory Committee on Vaccination and Immunization Practices of the Indian Academy of Pediatrics. Indian Pediatr. 2019;56:1041-8.

3. Ibanez M, Casas AA, Martinez CO, Aguado EJ, Mateos MMA. Humoral immunity in pediatric patients with acute lymphoblastic leukemia. Allergol Immunol Pathol (Madr). 2003;31:303-10.

4. Ljungman P. Vaccination of immune compromised hosts. In: Plotkin SA, Orenstein WA, Offit PA, Edwards KM, editors. Vaccines. $7^{\text {th }}$ ed. Philadelphia: Elsevier; 2018. p.1355-69.

5. Rubin LG, Levin MJ, Ljungman P, Davies EG, Avery R, Tomblyn M, et al. 2013 IDSA clinical practice guideline for vaccination of the immunocompromised host. Clin Infect Dis. 2014;58:e44-100.

6. Centers for Disease Control and Prevention. General Best Practice Guidelines for Immunization for Altered Immunocompetence Situations by Advisory Committee on Immunization Practices (ACIP). Available from: https:/ /www.cdc.gov/vaccines/hcp/acip-recs/general-recs/ immunocompetence.html. Accessed October 25, 2019.

7. Zignol M, Peracchi M, Tridello G, Pillon M, Fregonese F, D'Elia $\mathrm{R}$, et al. Assessment of humoral immunity to poliomyelitis, tetanus, hepatitis $\mathrm{B}$, measles, rubella, and mumps in children after chemotherapy. Cancer. 2004;101:635-41.

8. Fioredda F, Plebani A, Hanau G, Haupt R, Giacchino M, Barisone $\mathrm{E}$, et al. Re-immunisation schedule in leukaemic children after intensive chemotherapy: a possible strategy. Eur J Haematol. 2005;74:20-3.

9. Laws HJ, Calaminus G, Göbel U. Assessment of humoral immunity to poliomyelitis, tetanus, hepatitis B, measles, rubella, and mumps in children after chemotherapy. Cancer. 2005;103:1759.

10. Cecinati V. Vaccinations in Children with Cancer. Available from: file:///G:/sanjay\%20DOCUMENTS/sanjay\%20 Publi cations/viii.\%20Dr\%20Deepak/guidelines/Ch37-Vacci nation\%20Master.pdf. Accessed November 15, 2019.

11. Meisel R, Toschke M, Heilingensetzer C, Dilloo D, Laws HL, Kries RV. Increased risk for invasive pneumococcal diseases in children with acute lymphoblastic leukaemia. $\mathrm{Br}$ J Haematol. 2007;137:457-60.

12. Reinhardt D, Houliara K, Pekrun A, Lakomek M, Krone B. Impact of conventional chemotherapy on levels of antibodies against vaccine-preventable diseases in children treated for cancer. Scand J Infect Dis. 2003;35:851-7. 